\title{
Endothelial dysfunction in metabolic syndrome patients with and without hypertension
}

\begin{abstract}
The objective is to investigate if patients with metabolic syndrome have a difference in endothelial function (EF) and functional aerobic capacity as they were normotensive or hypertensive. Sixty-three consecutive primary prevention patients who came for the first time to a metabolic clinic, were studied with stress test and flow mediated dilation (FMD). The study was blind for the crossover results. Mean population age was 52 years and $60 \%$ among them were men. Ninety percent of patients accomplished metabolic syndrome criteria (by International Diabetes Federation criteria). There was no a significant difference in functional aerobic capacity between normotensive and hypertensive patients, despite it was calculated by METS achieved in the stress test (median 5.81 and $4.63 \mathrm{p}=0.114$ ) or by percent of capacity predicted by gender and age (median $79.7 \%$ and $63.2 \% \mathrm{p}=0.243$ ). In the whole population we found a significant difference in EF between normotensive and hypertensive (median $10 \%$ and $8 \% \mathrm{p}=0.002$ ) and between patients without and with metabolic syndrome (median $11 \%$ and $8 \% \mathrm{p}=0.023$ ). In the group of patients with metabolic syndrome we found also a significant difference in EF between normotensive and hypertensive (median $10 \%$ and $7.5 \% \mathrm{p}=0.006$ ) but we did not find a significant difference in functional aerobic capacity. Endothelial function was associated to hypertension and waist in multiple linear regression model. We conclude that in this population patients with metabolic syndrome and hypertension have poorer endothelial function than patients with metabolic syndrome and normotension.
\end{abstract}

Keywords: metabolic syndrome, hypertension, endothelial function, functional aerobic capacity
Volume 13 Issue 3 - 2020

\author{
Raúl Pisabarro Garcia,' Carlos Bermúdez,' \\ José Aníbal Manfredi, ${ }^{2}$ Mauricio Gutierrez' \\ 'Department of Endocrinology and Metabolism, Universidad de \\ la República Oriental del Uruguay, Uruguay \\ ${ }^{2}$ Department of Cardiology, Universidad de la República \\ Oriental del Uruguay, Uruguay
}

Correspondence: Raúl Pisabarro Garcia, Department of Endocrinology and Metabolism, Universidad de la República Oriental del Uruguay, Uruguay, Tel 005989962418I,

Email rpisabarro@pisabarro.com

Received: April 16, 2020 | Published: May 18, 2020
Abrréviations: FMD, flow-mediated vasodilation; ED, endothelial dysfunction; EF, endothelial function; MS, metabolic syndrome

\section{Introduction}

Atherosclerotic diseases are the leading cause of death in developed countries. Endothelial cells play a critical regulatory role in vascular health, including vascular smooth muscle cell relaxation and growth inhibition, inhibition of inflammatory responses, and antithrombotic actions. Endothelial dysfunction is pivotal to development of atherosclerosis. It is present in the earliest stages (e.g., preceding angiographic or ultrasonic evidence of obstructive plaque) as well as in later stages of arterial disease, contributing to clinical sequelae related to tissue damage (e.g., ischemia, infarction, and organ failure). Metabolic syndrome (MS) is a combination of several cardio metabolic risk factors including obesity, impaired glucose tolerance, high blood pressure, and dyslipidemia. Although controversy has surrounded both the pathophysiological basis and the clinical utility of the metabolic syndrome, it has been associated with an increased risk of cardiovascular disease.

The cardiorespiratory fitness-mortality risk association is robust and inverse, regardless age, race, gender, documented cardiovascular disease or comorbidities. ${ }^{1-3}$ Blood pressure is an important determinant of the risk of cardiovascular disease in the general population, and it is well established that interventions that lower blood pressure prevent cardiovascular events. ${ }^{4}$ The objective of this study is to investigate if patients with metabolic syndrome have a difference in endothelial function and functional aerobic capacity as they were normotensive or hypertensive.

\section{Methods}

We studied sixty-three consecutive patients in primary prevention patients who came for the first time to a metabolic clinic. Characteristics of the entire population (Table 1); the mean age was 52years. All the patients were white, $40 \%$ were women, $90 \%$ accomplished metabolic syndrome criteria (by International Diabetes Federation criteria), 48\% had normal glycaemia, $11 \%$ type 2 diabetes, $63.5 \%$ were hypertensive and $31.8 \%$ were obese. There were no significant differences between normotensives and hypertensives in sex, race, BMI, metabolic syndrome, diabetes, sedentary life style, LDL and HDL cholesterol, triglycerides and statins use. Hypertensives patients were older (mean age 55years vs. 46years $\mathrm{p}=0.012$ ) and had a higher Score Risk (mean 1.0 vs. $0.5 \mathrm{p}<0.001)$ (Table 2).

In this group of patients with metabolic syndrome there are no significant differences between normotensives and hypertensives, except older age and a higher Score Risk in hypertensives. We conducted a stress test and flow mediated dilatation of the brachial artery for endothelial function (EF). The studies were blinded for the crossover results. Each patient underwent a symptom-limited maximal exercise testing. The tests were stopped if the patient requested to stop due to exhaustion, or the physician stopped the test due to medical reasons. The physicians stopped the test either due to symptoms or positive high-risk features, including a drop in systolic $\mathrm{BP}$ of $>20 \mathrm{~mm} \mathrm{Hg}$ with increasing workload, high-risk ST-changes, 
and sustained ventricular tachycardia. The parameters recorded during the stress test were: symptoms, continuous heart rate and ECG, and every 3 minutes BP recordings. The patients were monitored during recovery for symptoms, vital signs, and ECG for the first 3 minutes if they were stable, or longer if any abnormalities were noted. Brachial flow-mediated endothelial-dependent dilation (FMD) is a validated physiologic measure of the endothelial function. ${ }^{5}$

Table I Characteristics of the entire population

\begin{tabular}{llll}
\hline & Normotensives & Hypertensives & $\mathbf{p}$ \\
\hline $\mathbf{n}$ & 20 & 43 & \\
\hline Female (\%) & $\mathrm{II}$ & 14 & 0.09 \\
Age (years) & 46 & 55 & 0.012 \\
White race (n) & 20 & 43 & 0.126 \\
BMI & 29 & 33 & 0.126 \\
MS (n) & 17 & 40 & 0.372 \\
Diabetes (n) & 1 & 6 & 0.415 \\
Sedentary life style & 9 & 29 & 0.09 \\
LDL (mg/dl) & 122 & 116 & 0.495 \\
HDL (mg/dl) & 53 & 46 & 0.978 \\
Triglycerides(mg/dl) & 134 & 138 & 0.598 \\
Statins use (n) & 6 & 19 & 0.284 \\
Score Risk & 0.5 & 1 & $<0.001$ \\
\hline
\end{tabular}

Table 2 The group of patients with MS had these characteristics

\begin{tabular}{llll}
\hline & Normotensives & Hypertensives & p \\
\hline $\mathbf{N}$ & $\mathbf{1 7}$ & $\mathbf{4 0}$ & \\
\hline Female (\%) & 8 & 12 & 0.217 \\
Age (years) & 46 & 55 & 0.012 \\
White race (n) & 17 & 40 & 0.126 \\
BMI & 30 & 33 & 0.294 \\
Diabetes (n) & 1 & 6 & 0.662 \\
Sedentary life style & 9 & 28 & 0.217 \\
LDL (mg/dl) & 122 & 116 & 0.502 \\
HDL (mg/dl) & 50 & 46 & 0.903 \\
Triglycerides(mg/dl) & 140 & 141.5 & 0.807 \\
Statins use (n) & 6 & 18 & 0.497 \\
Score Risk (\%) & 0.5 & 1 & $<0.001$ \\
\hline
\end{tabular}

Assessment of FMD were performed by imaging the left brachial artery at the distal third of the upper arm using a $7 \mathrm{MHz}$ transducer.
The diameter of the brachial artery was measured at baseline, as the average resulting from five measurements. After measuring resting artery diameter, a blood pressure cuff was inflated below the elbow (distal to imaged artery segment) surpassing systolic blood pressure by $50 \mathrm{mmHg}$ for five minutes. Brachial artery diameter was measured after cuff release during reactive hyperemia to determine peak dilation. In the first 15 seconds of the arterial occlusion release, the mean velocity of the brachial artery was measured. Between the first minute and the four minutes of decompression the diameter of the brachial artery was measured every 30 seconds. The peak post-decompression diameter corresponded to the maximum of all diameters measured in that period.

Flow-mediated vasodilation (FMD) was determined as the ratio between the peak decompression diameter, minus the basal diameter, divided by the basal diameter. Endothelial dysfunction (ED) was diagnosed if the FMD was less than $10 \%$. All measurements were performed in the morning after a 12-h overnight fast and at least 48 $\mathrm{h}$ after the last exercise session, in a quiet, temperature-controlled environment. Nitroglycerin was not administered in this protocol to avoid the occurrence of headache and hypotension in ambulatory and fasting patients. The stress test and the study of FMD were performed on different days and by different researchers who did not know the results of previous tests.

\section{Statistical analysis}

In the descriptive analysis summary measures of central tendency and frequencies were used. In the inferential bivariate analysis, continuous variables were studied: Anderson test to verify normal distribution, Barttlet test to check homogeneity of groups, ANOVA or Kruskal-Wallis as applicable, for comparison of central tendency. In the comparison of categorical and dichotomous variables, a chisquare test by the Mantel-Haenszel was conducted; when the expected values were less than 5, Fischer's exact test was used. Multiple linear regression model was used for FMD. For statistical processing, the package R 3.3.2 and Microsoft Excel was used.

\section{Results}

In the whole population there was no significant difference in functional aerobic capacity between normotensive and hypertensive patients by METS achieved (median 5.81 and $4.63 \mathrm{p}=0.114$ ). If we use the percent of functional aerobic capacity predicted by gender and age, there was no statistically significant difference (median 79.7\% and $63.2 \% \mathrm{p}=0.243$ ). In the whole population we found a significant difference in endothelial function measured by FMD between normotensive and hypertensive (median $10 \%$ and $8 \% \mathrm{p}=0.002$ ) and between patients without and with metabolic syndrome (median $11 \%$ and $8 \% \mathrm{p}=0.023$ ). In the group of patients with metabolic syndrome we did not find a significant difference in functional aerobic capacity between normotensive and hypertensive patients by METS achieved ( 5.81 and $4.63 \mathrm{p}=0.114$ ) or by percent of functional aerobic capacity predicted $(78 \%$ vs. $63 \% \mathrm{p}=0.443)$. Finally, in this group we found also a significant difference in FMD between normotensive and hypertensive (median 10\% and $7.5 \% \mathrm{p}=0.006$ ) (Figure 1). By multiple linear regression model, flow mediated dilation were significantly associate with two parameters: hypertension and waist (r2 0,2906), independently of age, gender, triglycerides, HDL and LDL cholesterol, glycemia and use of statins (Table 3). 


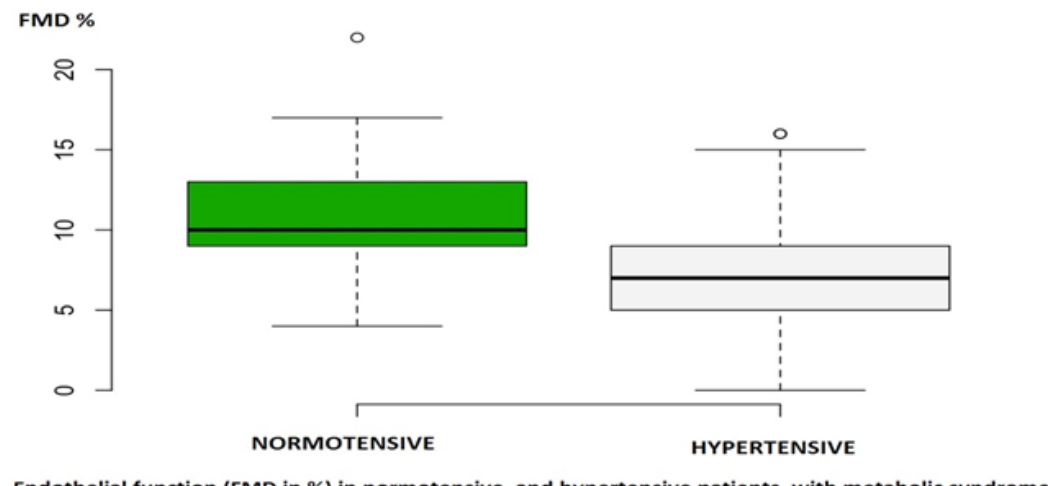

Figure I Endothelial Function by FMD in patients with metabolic syndrome according they were normotensives (green) or hypertensives (gray).

Table 3 Multiple linear regression model for flow mediated dilation (FMD \%)

\begin{tabular}{lllll}
\hline & Estimate & St Error & t value & $\operatorname{Pr}(>t)$ \\
\hline Intercept & 24.263027 & 5.471645 & 4.434 & $4.57 \mathrm{e}-05^{* * *}$ \\
HDL cholesterol & 0.011414 & $0.05206 \mathrm{I}$ & 0.219 & 0.8273 \\
Triglycerides & -0.007770 & 0.005826 & -1.334 & 0.1879 \\
Hypertension & -2.889699 & 1.177727 & -2.454 & $0.0174 *$ \\
Glycemia & -0.588459 & 0.740990 & -0.794 & 0.4306 \\
Waist & -0.084822 & $0.03845 \mathrm{I}$ & -2.206 & $0.0317^{*}$ \\
Male gender & -1.512587 & 1.282632 & -1.179 & 0.2435 \\
Age & 0.006969 & $0.05201 \mathrm{I}$ & 0.134 & 0.8939 \\
LDL cholesterol & -0.021637 & 0.012305 & -1.758 & 0.0843 \\
* $\left.^{*}<0.05\right)$. & & & &
\end{tabular}

Residual standard error: 3.852 on 62 degrees of freedom. Multiple $r^{2}: 0.3821$, Adjusted $r^{2}: 0.2906$

\section{Discussion}

Endothelial dysfunction is a well established response to cardiovascular risk factors and precedes the development of atherosclerosis. ${ }^{5,6}$ When cardiovascular risk factors are treated, the endothelial dysfunction improved. It is also an independent prognosticator of cardiac events. In subjects with hypertension, beyond the increased blood pressure in the arteries that shows the sphygmomanometer, we can often detect other disorders: endothelial dysfunction, increased peripheral vascular resistance and arterial stiffness among others. The metabolic syndrome, which is regarded as a prediabetic state, is characterized by a concurrence of interrelated cardiovascular risk factors, including abdominal obesity, insulin resistance, hypertension, dyslipidemia, and glucose intolerance7. Endothelial dysfunction is common in the metabolic syndrome. ${ }^{8}$

Flow mediated dilation of the brachial artery is a very useful method for measuring EF. In a nationwide multicenter study they found that the results of FMD were accurate, with good reproducibility. ${ }^{9}$ Reactive hiperemia induced by shear stress produces the release of nitric oxide, which acts as a vasodilator. This response is attenuated in ED. ${ }^{10,11}$

Endothelial dysfunction, measured by FMD, is associated with increased risk for development of diabetes ${ }^{12}$ and cardiovascular morbidity and mortality in primary prevention patients according meta-análysis. ${ }^{13}$ In patients with history of coronary artery disease, better FMD was associated with lower risk of cardiovascular events. ${ }^{14}$

In metabolic syndrome patients, each one stándar deviation worsening in endothelial function measured by FMD was associated with doubled cardiovascular risk. ${ }^{15}$ Microvascular endotelial dysfunction, measured by peripheral arterial tonometry, was associated with two-fold increased risk of developing solid-tumor cancer. ${ }^{16}$ Metabolic syndrome, wich is associated to endothelial dysfunction, also increase the incidence of cancer.

There is a considerable body of evidence indicating that a good level of cardiorespiratory fitness reduces the risk of cardiovascular disease ${ }^{1-3}$ High levels of physical activity or fitness may be particularly beneficial to individuals with insulin-resistant conditions, such as the metabolic syndrome, type 2 diabetes, obesity or hypertension. ${ }^{4}$ The stress test is the most used method for measuring functional aerobic capacity. We studied it by METS achieved and by percent of the capacity predicted by gender and age. We investigated whether there were differences between hypertensive and normotensive with or without metabolic syndrome in endothelial function and aerobic capacity. In the whole population there were no differences in functional aerobic capacity between normotensives and hypertensives, possibly due to the low levels of physical activity performed by patients with high prevalence of overweight, obesity and low aerobic capacity.

Endothelial function, however, had significant differences across the normotensive or hypertensive populations, and according to whether they have or not metabolic syndrome, which would indicate an increased risk in these pathologies. Moreover, in patients with metabolic syndrome there was a significant alteration of the endothelial function, which was more pronounced with the association of hypertension. This finding is consistent with the work of Dell'Omo and col. in which hypertensive subjects with MS had the greatest level of endothelial dysfunction in comparison to hypertensive subjects without the MS and normotensives subjects. ${ }^{17}$

Score Risk, a prognostic indicator of cardiovascular events, also showed a significant difference between normotensive and hypertensive subjects in metabolic syndrome patients. In the present study, we measured endothelial function in normotensive and hypertensive patients with MS, and found in the latter group a significant association with worse endothelial function. These finding may reinforce the hypothesis of the role of ED in the development of hypertension also in patients with MS and the possible benefit of therapeutic measures to improve EF, both in the prevention and in 
the treatment of hypertension. By multiple linear regression analysis, waist and hypertension were significantly associated with VDMF. The waist is related to endothelial dysfunction and to visceral obesity. Visceral obesity is a paramount risk factor to the development of arterial hypertension. Endothelial dysfunction and visceral obesity are related to the development of hypertension. There is a close relation between endothelial dysfunction, metabolic syndrome and hypertension, especially through oxidative stress in the vascular endothelium. ${ }^{18,19}$

\section{Conclusion}

In conclusion, the group of patients with metabolic syndrome and hypertension showed poorer endothelial function than patients with metabolic syndrome and normotension. This fact may explain the worse cardiovascular outcome in patients with metabolic syndrome and hypertension, and the usefulness of measures to improve endothelial function for the prevention, treatment and prognosis of hypertension in these high risk patients.

\section{Acknowledgments}

None.

\section{Conflicts of interest}

The authors declare there are no conflicts of interest related to the article.

\section{Funding}

None.

\section{References}

1. US Department of Health and Human Services. 2008 Physical Activity Guidelines for Americans. Washington, DC: US Department of Health and Human Services; 2008.

2. Australian Government Department of Health. Australia's Physical Activity and Sedentary Behaviour Guidelines for Adults (18-64years). Canberra, Australia: Australian Government Department of Health; 2014.

3. Myers J, Prakash M, Froelicher V, et al. Exercise Capacity and Mortality among Men Referred for Exercise Testing. N Engl J Med. 2002;346:793801 .

4. Weber MA, Schiffrin EL, White WB, et al. Clinical Practice Guidelines for the Management of Hypertension in the Community A Statement by the American Society of Hypertension and the International Society of Hypertension. J Clin Hypertens (Greenwich). 2014;16(1):14-26.

5. Widlansky ME, Gokce N, Keaney JF, et al. The clinical implications of endothelial dysfunction. J Am Coll Cardiol. 2003;42(7):1149-1160.
6. Landmesser U, Hornig B, Drexler H. Endothelial function: a critical determinant in atherosclerosis? Circulation. 2004;109(21 Suppl 1):II27II 33.

7. Redon J, Cifkova R, Laurent S, et al. Mechanisms of hypertension in the cardiometabolic syndrome. J Hypertension. 2009;27(3):441-451.

8. Melikian N, Chowienczyk P, MacCarthy PA, et al. Determinants of endothelial function in asymptomatic subjects with and without the metabolic syndrome. Atherosclerosis. 2007;197(1):375-382.

9. Ghiadoni L, Faita F, Salvetti M, et al. Assessment of flow-mediated dilation reproducibility: a nationwide multicenter study. $J$ Hypertens. 2012;30(7):1399-1405.

10. Corretti MC, Anderson TJ, Benjamin EJ, et al. Guidelines for the ultrasound assessment of endothelial-dependent flow-mediated vasodilatation of the brachial artery: a report of the International Brachial Artery Reactivity Task Force. J Am Coll Cardiol. 2002;39(2):257-265.

11. Deedwania PC. Mechanisms of endothelial dysfunction in the metabolic syndrome. Curr Diab Rep. 2003;3:289-292.

12. Rossi R, Cioni E, Nuzzo A, et al. Endothelial-Dependent Vasodilation and Incidence of Type 2 Diabetes in a Population of Healthy Postmenopausal Women. Diabetes Care. 2005;28(3):702-707.

13. Matsuzawa Y, Kwon TG, Lennon RJ, et al. Prognostic Value of FlowMediated Vasodilation in Brachial Artery and Fingertip Artery for Cardiovascular Events: A Systematic Review and Meta-Analysis. $J$ Am Heart Assoc. 2015;4(11).

14. Maruhashi T, Soga J, Fujimura N, et al. Endothelial Dysfunction, Increased Arterial Stiffness, and Cardiovascular Risk Prediction in Patients With Coronary Artery Disease: FMD-J (Flow-Mediated Dilation Japan) Study A. J Am Heart Assoc. 2018;7(14).

15. Ryliškyte L, Navickas R, Šerpytis P, et al. Association of aortic stiffness, carotid intima-media thickness and endothelial function with cardiovascular events in metabolic syndrome subjects. Blood Press. 2019;28(2):131-138.

16. Toya T, Sara JD, Corban MT, et al. Assessment of peripheral endothelial function predicts future risk of solid-tumor cancer. Eur J Prev Cardiol. 2020;27(6):608-618

17. Dell'Omo G, Penno G, Pucci L, et al. Abnormal capillary permeability and endothelial dysfunction in hypertension with metabolic syndrome. Atherosclerosis. 2004;172(2):383-389.

18. Chaudhary P, Pandey A, Azad CS, et al. Association of oxidative stress and endothelial dysfunction in hypertension. Anal Biochem. 2020;590:113525.

19. Bond V Jr, Becknel K, Kumar K, et al. Association of Endothelial Function with Parental Hypertension in Normotensive-Obese AfricanAmerican Women: A Pilot Study. Adv Prev Med. 2019;2019:5854219. 